\title{
Estimación de la curva de crecimiento en machos bubalinos en una empresa cubana
}

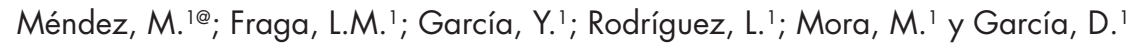 \\ Instituto de Ciencia Animal. San José de las Lajas. Mayabeque. Cuba.
}

\section{PALABRAS CLAVE ADICIONALES}

Búfalo.

Curva de crecimiento.

Modelos no lineales.

\section{RESUMEN}

Para la caracterización de la curva de crecimiento se utilizaron los datos de 11 pesajes mensuales (1 188 observaciones), desde los 8 hasta los 18 meses de edad correspondientes a 108 búfalos de la raza mestizo de Bufalipso que se encontraban en prueba de comportamiento entre los años 2007 y 2012, los cuales vinieron de 16 rebaños localizados en una empresa de la región occidental de Cuba. Los pesos individuales de cada animal fueron ajustados a las edades que correspondieron a cada uno de los pesajes, y además se utilizó un modelo mixto para corregir los mismos a los efectos mes y año de destete. En la estimación de la curva de crecimiento y sus parámetros $\left(\beta_{0}, \beta_{1}\right.$ y $\left.\beta_{2}\right)$ de los animales se utilizaron los modelos Brody, Gompertz, Von Bertalanffy y Logístico. La bondad de ajuste y discriminación entre los modelos utilizados demostraron un alto ajuste en los cuatros modelos, con $\mathrm{R}^{2}$ por encima de $90 \%$. El modelo Logístico fue el de mejor ajuste para describir la curva de crecimiento en machos bubalinos mestizos Bufalipso según los criterios estadísticos evaluados bajo estas condiciones.

\section{Growing curve estimation of bubaline males in a Cuban enterprise}

\section{SUMMARY}

To characterize the growing curve it was utilized a database with 11 monthly weighting (1188 records) from 8 to 18 months of age corresponding to 108 graded male buffaloes in performance tests between the 2007 and 2012 control years which came from 16 herds located in an enterprise at the west region of Cuba. The individual weights of each animal were adjusted to their corresponded weighting age then it was employed after a mixed model to adjust them to weaning month and year. To estimate the growing curves and their parameters $\left(\beta_{0}, \beta_{1}\right.$ and $\left.\beta_{2}\right)$ were utilized the Brody, Gompertz, Von Bertanlaffy and Logistic models. The goodness of fit and discrimination between models demonstrated a high adjustment to models evaluated with $\mathrm{R}^{2}$ higher than $90 \%$. The Logistic model obtained the best adjustment to describe the growing curve of the male graded Buffalypso according to the statistic criterion considered under these conditions.

\section{INFORMACIÓN}

Cronología del artículo.

Recibido/Received: 1.7.2014

Aceptado/Accepted: 29.1.2015

On-line: 10.6 .2015

Correspondencia a los autores/Contact e-mail:

mmendez@ica.co.cu

\section{INTRODUCCIÓN}

El interés sobre los búfalos surge al ser reconocidas sus bondades en la producción de leche, carne y labores de trabajo, haciendo uso de recursos alimenticios y ocupando espacios que no son apropiados para otras especies (Oliveira et al., 1991).

Varios estudios reportaron al búfalo como un animal precoz para producir carne en sistemas de pastoreo (Amorin et al., 1999). Al compararlos con ganado vacuno se ha encontrado que tienen mayores tasa de crecimiento alcanzando el peso para el sacrificio en periodos más cortos (Angulo et al., 2006), una de las explicaciones que se encuentra al respecto es la capacidad que tiene la especie de aprovechar con mayor eficiencia las pasturas, presentando mejores tasas de conversión alimenticia (Vale et al., 2002).

El crecimiento es una de las características que debe ser mejorada, dada su importancia económica en los sistemas de producción de carne, ya que se relaciona con características productivas y reproductivas (Blasco, 2004). Se espera que los animales que crecen más rápido, en términos de peso corporal, puedan iniciar el funcionamiento fisiológico de la reproducción y producción de leche más temprano.

Debido a la gran cantidad de datos generados durante el crecimiento, pueden surgir dificultades de interpretación, por lo que el uso de modelos estadísticos adecuados, permite resumirlos en pocos parámetros 
de acuerdo al modelo utilizado (Val et al., 2004). En general, el crecimiento animal es explicado por modelos como el logístico, Brody, Gompertz, Richards, entre otros (Blasco, 2004). Además, se ha hecho uso de funciones lineales, y polinómicas entre otras, para tratar de predecir el peso adulto o el grado de madurez sin tener que realizar mediciones repetidas o esperar que el animal alcance su madurez para tomar decisiones relacionadas con la producción (Moore, 1985).

La medida de crecimiento más usual es la medición del peso corporal. En este sentido, el crecimiento puede definirse a través de la curva de crecimiento total o de ganancia acumulativa de peso que expresa el crecimiento como un aumento que se va acumulando durante un período de tiempo prefijado (Bavera et al., 2005).

Las curvas del crecimiento reflejan la relación entre la edad del animal y el impulso propio del individuo para crecer y madurarse en su conjunto corporal y el ambiente en el cual estos impulsos están expresados, por lo que su conocimiento es importante en el monitoreo de un hato, ya que ellas expresan gran cantidad de información inherentes al animal durante toda su vida. Por lo tanto, la asociación de esas estimativas a las características productivas genera datos que permiten el conocimiento de las exigencias del animal (Fitzhugh, 1976).

En Cuba, la caracterización de las curvas de crecimiento en búfalos mestizos no es amplia, solamente se disponen de pocas investigaciones (Fundora et al., 2006; Arias et al., 2010 y Torres et al., 2012). Por lo que el objetivo del presente estudio consistió en estimar la curva de crecimiento de búfalos procedentes de la granja Majana de la EPG Los Naranjos, en Artemisa, a través de cuatro modelos matemáticos no lineales.

\section{MATERIAL Y MÉTODOS}

Se utilizaron los datos de 11 pesajes mensuales (1188 observaciones), desde los 8 hasta los 18 meses de edad correspondientes a 108 búfalos de la raza mestizo de Bufalipso que se encontraban en prueba de comportamiento entre los años 2007 y 2012, los cuales vinieron de 16 rebaños localizados en una empresa de la región occidental de Cuba. Los animales fueron criados en pastizales naturales y complementados con forrajes, agua ad libitum y sombra natural.

Los pesos individuales de cada animal fueron ajustados a las edades que correspondieron a cada uno de los pesajes con la utilización de la formula:

$$
\text { Pajustado }=\mathrm{P}_{\mathrm{i}}+\left(\left(\mathrm{P}_{\mathrm{i}}-\mathrm{P}_{0}\right) / \text { edad }_{\mathrm{i}}\right) *\left(\mathrm{n}-\mathrm{edad}_{\mathrm{i}}\right)
$$

Donde:

Pajustado $=$ peso individual ajustado.

$\mathrm{P}_{\mathrm{i}}=$ valor del i-ésimo pesaje.

$\mathrm{P}_{0}=$ valor del pesaje anterior.

$\mathrm{n}=$ constante que representa la media de la edad para $\mathrm{P}_{\mathrm{i}}$.

edad $_{1}=$ edad para $\mathrm{P}_{\mathrm{i}}$.
El modelo mixto utilizado (PROC MIXED del SAS, 2007, Versión 9.1.3) fue el siguiente:

$$
\mathrm{y}_{\mathrm{ijk}}=\mu+\alpha_{\mathrm{i}}+\beta_{\mathrm{j}}+\gamma_{\mathrm{k}}+\mathrm{a}(\alpha)_{\mathrm{i}}+\mathrm{e}_{\mathrm{ijk} \mathrm{l}}
$$

Donde:

$\mathrm{y}_{\mathrm{ijk}}=$ valor para cada una de las observaciones de los indicadores evaluados.

$\mu=$ Media de la población.

$\alpha_{\mathrm{i}}=$ Efecto fijo de rebaño $(\mathrm{i}=1,2, \ldots, 6)$.

$\beta_{\mathrm{j}}=$ Efecto fijo de año de destete $(\mathrm{i}=1, \ldots, 5)$.

$\gamma_{\mathrm{k}}=$ Efecto fijo de mes de destete $(\mathrm{i}=1, \ldots, 7)$.

$\mathrm{a}(\boldsymbol{\alpha})_{\mathrm{i}}=$ Efecto aleatorio del arete de cada animal 1 anidado dentro de cada rebaño $i$.

$\mathrm{e}_{\mathrm{ijk}}=$ error residual, normal e independientemente distribuido $\left(0, \sigma^{2}\right)$.

Para estimar la curva de crecimiento y sus parámetros $\left(\beta_{0}, \beta_{1}\right.$ y $\left.\beta_{2}\right)$ de los animales se utilizaron los modelos Brody, Gompertz, Von Bertalanffy y Logístico (tabla I) a través del procedimiento NLIN (SAS, 2007).

Para seleccionar el modelo de mejor ajuste fueron considerados los criterios estadísticos propuestos por Torres et al. (2012) empleando el paquete Statgraphics Plus 5.1 (2000).

Tabla I. Modelos no lineales para describir la curva de crecimiento en búfalos (Nonlinear models to describe the growth curve in buffalo).

\begin{tabular}{ll}
\hline Brody & $y(t)=\beta_{0}\left(1-\beta_{1} \exp \left(\beta_{2} t\right)\right)+e$ \\
Von Bertanlaffy & $y(t)=\beta_{0}\left(1-\beta_{1} \exp \left(-\beta_{2} t\right)\right)^{3}+e$ \\
Gompertz & $y(t)=\beta_{0} \exp \left(-\beta_{1} \exp \left(-\beta_{2} t\right)\right)+e$ \\
Logístico & $\left.y(t)=\beta_{0}+b_{0}\right)\left(1+\beta_{1} \exp \left(\beta_{2} t\right)^{-1}+e\right.$ \\
\hline
\end{tabular}

\section{RESULTADOS Y DISCUSIÓN}

Los valores de los parámetros de las curvas estimadas se muestran en la tabla II. El mayor valor obtenido para el parámetro $\beta_{0}$ correspondió al modelo de Brody $(286 \pm 9 \mathrm{~kg})$ y el menor el Logístico $(263 \pm 6 \mathrm{~kg})$.

Este resultado es similar a lo reportado por Torres et al. (2012) en un estudio realizado con 15 hembras bubalinas de la misma raza que se encontraban en pastoreo, donde hubo sobreestimación de este parámetro para el modelo de Brody $(1053,33 \pm 68,74 \mathrm{~kg})$. En Brasil Malhado et al. (2008) al utilizar pesajes desde el nacimiento hasta los 40 meses de edad de 236 búfalos (machos y hembras) de la raza Murrah, criados a pasto en la región Nordeste de Brasil con alimentación suplementaria en la época de la sequía y pertenecientes al Programa de Mejora Genética de Bufalinos (Promebul), se pudo verificar que el mayor valor fue obtenido con el modelo Brody $(688,0 \pm 4,6 \mathrm{~kg})$ y el menor, el de la curva logística $(601,9 \pm 2,3 \mathrm{~kg})$.

Según Silva et al. (2004) otro parámetro importante es el parámetro $\beta_{2}$, que se conoce como índice de madurez y determina la eficiencia del crecimiento de un animal. Con la estimación de este parámetro ocurre lo contrario que con el parámetro $\beta_{0}$, el valor más alto correspondió al modelo Logístico $(0,01 \pm 0,002)$, mientras 
Tabla II. Estimaciones de los parámetros $\left(\beta_{0}, \beta_{1} \mathrm{y}\right.$ $\beta_{2}$ ) para los modelos no lineales evaluados (Parameter estimates $\left(\beta_{0}, \beta_{1}\right.$ and $\left.\beta_{2}\right)$ for non-linear models evaluated).

\begin{tabular}{lccc}
\hline Modelos & \multicolumn{3}{c}{ Parámetros } \\
\hline & $\beta_{0}$ & $\beta_{1}$ & $\beta_{2}$ \\
Brody & $286 \pm 9$ & $1,8 \pm 1,0$ & $0,007 \pm 0,002$ \\
Von Bertanlaffy & $276 \pm 7$ & $0,8 \pm 0,5$ & $0,008 \pm 0,002$ \\
Gompertz & $272 \pm 7$ & $2,9 \pm 1,6$ & $0,009 \pm 0,002$ \\
Logístico & $263 \pm 6$ & $4,8 \pm 2,8$ & $0,01 \pm 0,002$ \\
\hline
\end{tabular}

$\beta_{0}=$ Peso asintótico; $\beta_{1}=$ Constante de integración; $\beta_{2}=$ Tasa de madurez.

Tabla III. Criterios de bondad de ajuste para los modelos analizados (Goodness of fit criteria for the models analyzed).

\begin{tabular}{|c|c|c|c|c|}
\hline \multirow{2}{*}{ Criterios de ajuste } & \multicolumn{4}{|c|}{ Modelos } \\
\hline & Brody & Von Bertanlaffy & Gompertz & Logístico \\
\hline $\begin{array}{l}\text { Coeficiente de } \\
\text { determinación }\left(R^{2}\right)\end{array}$ & 90,38 & 91,64 & 93,76 & 96,12 \\
\hline $\begin{array}{l}\text { Cuadrado medio del } \\
\text { error de predicción } \\
\text { (CMEP) }\end{array}$ & 38,70 & 37,21 & 36,50 & 34,42 \\
\hline $\begin{array}{l}\text { Error estándar de } \\
\text { estimación }\end{array}$ & 7,22 & 7,02 & 6,04 & 5,87 \\
\hline \multicolumn{5}{|l|}{ Análisis de residuos } \\
\hline $\begin{array}{l}\text { Error absoluto } \\
\text { medio (EAM) }\end{array}$ & 4,32 & 4,27 & 4,24 & 3,89 \\
\hline $\begin{array}{l}\text { Porcentaje de error } \\
\text { absoluto medio } \\
\text { (PEAM) }\end{array}$ & 1,52 & 1,50 & 1,48 & 1,42 \\
\hline
\end{tabular}

que para los restantes modelos oscilaron entre $0,007 \mathrm{y}$ 0,009. Torres et al. (2012) Araújo et al. (2012) en Cuba y Brasil respectivamente reportaron comportamiento similar.

Los ajustes a través de los diferentes modelos (tabla III) aportan coeficientes de determinación $\left(\mathrm{R}^{2}\right)$ altos, que variaron entre $90,38 \%$ y $96,12 \%$, siendo superior en el modelo Logístico. El cuadrado medio del error de predicción (CMEP) inferior correspondió también al modelo Logístico $(34,42)$, aumentado entre un $2 \%$ a un $9 \%$ para los otros tres modelos probados. Comportamiento similar se obtuvo con el error estándar de la estimación $(5,87)$ y para el análisis de residuos, evaluado este último a través de 2 criterios estadísticos el error absoluto medio (EAM) y el porcentaje de error absoluto medio (PEAM) con valores de 3,89 y 1,42 respectivamente que correspondieron al modelo Logistico.

Diversos autores (Malhado et al., 2008, Torres et al., 2012, Araújo et al., 2012) en investigaciones realizadas en la especie bubalina aun utilizando otros criterios estadísticos para la elección del modelo de mejor ajuste el resultado fue muy similar al aquí obtenido, con la utilización de diferentes razas y distintas formas de manejo y alimentación. Sin embargo, los resultados no coinciden con los obtenidos por Arias et al. (2010) y Agudelo-Gómez et al. (2009), los cuales utilizaron
Tabla IV. Correlaciones entre los parámetros $\beta_{0} \mathrm{y}$ $\beta_{2}$ de los modelos no lineales evaluados (Correlations between the parameters $\beta_{0}$ and $\beta_{2}$ evaluated non-linear models).

\begin{tabular}{lc}
\hline Modelos & Correlaciones \\
\hline Brody & $-0,99$ \\
Von Bertanlaffy & $-0,98$ \\
Gompertz & $-0,98$ \\
Logístico & $-0,96$ \\
\hline
\end{tabular}

otros modelos no lineales a los aquí empleados por lo en sus investigaciones concluyeron que el polinomio de Legendre de orden 4 y el modelo de Brody para describir la curva de crecimiento de los animales respectivamente.

Las correlaciones entre los parámetros $\beta_{0} \mathrm{y} \beta_{2}$ de los cuatros modelos evaluados se muestran en la tabla IV, las cuales fueron negativas y altas, que animales con mayores tasas de crecimiento tienen menor probabilidad de alcanzar mayores pesos en la madurez, comparados con aquellos que crecen más despacio en el inicio de la vida. Se debe resaltar que esta relación, biológicamente, es la más importante (Sarmento et al., 2006).

Resultados similares fueron determinados por Malhado et al. (2008) en Brasil y por Arias et al. (2010) en Cuba donde obtuvieron valores de correlación de $-0,71$ y de $-0,99$ entre el peso adulto $\left(\beta_{0}\right)$ y la velocidad de crecimiento $\left(\beta_{2}\right)$ correspondiendo en ambos casos al modelo logístico.

\section{CONCLUSIONES}

El modelo logístico presentó el mejor ajuste para describir el crecimiento de los bufalinos estudiados, seguido de los modelos Gompertz, Von Bertalanffy y Brody bajo nuestras condiciones según los criterios estadísticos evaluados, por lo que se recomienda continuar estos estudios con mayor cantidad de animales incluyendo en los modelos efectos aleatorios, con el objetivo de seleccionar los mejores animales de mayor crecimiento.

\section{AGRADECIMIENTOS}

Se agradece a los compañeros de la empresa de donde se recogieron los datos y a todos aquellos que hicieron posible la realización de este trabajo.

\section{BIBLIOGRAFÍA}

Agudelo-Gómez, D.; Hurtado, N. and Cerón, M. 2009. Growth curves and genetic parameters in colombian buffaloes (Bubalus bubalis, Artiodactyla, Bovidae). Rev Colomb Cienc Pecu, 22: 178-188.

Amorin, A.; Hipólito, V.; Tonhati, B. e Baruselli, S. 1999. Melhoramento genético dos búbalinos. Simposio paulista de bubalinocultura. FFUNEP. Jaboticabal. pp. 27-50.

Angulo, R.; Agudelo-Gómez, D.; Cerón-Muñoz, M.F. and JaramilloBotero, S. 2006. Genetic parameters in buffalo calves fed at full milk in beef production system in middle Magdalena region of Colombia. Livest Res Rural Develop, 18: Article \#180. http://www.lrrd.org/ Irrd18/12/angu 18180.htm (15/05/2010).

Araújo, R.; Righetti, C.; Florisbal, M.C.; del Valle,A.; Gunski, R.J.; Everling, D.M. e Nogara, P.R. 2012. Modelos não-lineares clássicos párrafo 
descrever una curva de crescimento de búfalos da raça Murrah. Rev Cien Rural, 42: 520-525.

Arias, Y.; González-Peña, D.; Montes, I. y Domínguez, A. 2010. Ciencia y Tecnología Ganadera, 4: 136-142.

Bavera, G.; Bocco, O.; Beguet, H. y Petryna, A. 2005. Crecimiento, desarrollo y precocidad. Cursos de Producción Bovina de Carne, FAV UNRC. http://www.produccion-animal.com.ar/informacion_tecnica/ exterior/05-crecimiento_desarrollo_y_precocidad.pdf (07/02/2007).

Blasco, A. 2004. Proc. XIV International Course on Animal Genetic Improvement. Universidad Politécnica de Valencia. Spain.

Brody, S. 1945. Biogenetics and growth. Reinhold Publishing Corporation. New York. 1023 pp.

Fitzhugh, J.H. 1976. Analysis of growth curves and strategies for altering their shape. J Anim Sci, 42:1036-1051.

Fundora, O.; Torres, V.; González, M.A. y Noda, A. 2006. Curva de crecimiento de búfalos del río. Rev Cubana Cienc Agric, 40: 419.

Gompertz, B. 1925. On the nature of the function expressive of the law of human mortality, and on new method of determining the value of life contingencies. Philos Trans R Soc Lond, 115: 513-585.

Malhado, C.H.; de Amorin, A.; Carneiro, P.; Souza, J.C.; Wechsler, F.S.; Ber, J.P.; Azevedo, M.Ry Sereno, J.R. 2008. Modelos no lineales para describir el crecimiento de bufalinos de la raza Murrah. Arh Zootec, 57: 497-503.

Moore, A.J. 1985. A mathematical equation for animal growth from embryo to adult. Anim Prod, 40: 441-453.

Oliveira, A.L.; Velloso, L. and Schalech, E. 1991. Carcass characteristics and yield of zebu steers compared with water buffalo. In: World Buffalo Congress, 3. Varna. Bulgaria. pp. 1019-1026.
Sarmento, J.L.R.; Regazzi, A.J. e Souza, W.H. 2006. Estudo da curva de crescimento de ovinos Santa Inês. Rev Bras Zootecn, 35: 435-442.

SAS. 2010. SAS User's guide: Statistics. Versión 9.3. SAS Institute. INC. Cary. N.C. USA

Silva, N.; Aquino, L.H.; Silva, F.F. e Oliveira, A. 2004. Curvas de crescimento e influência de fatores não-genéticos sobre as taxas de crescimento de bovinos da raça Nelore. Ciênc Agrotec, 28: 647-654.

Statgraphics 2000. Statgraphics Plus para Windows 5.1 Statistical Graphics Corporation.

Torres, V.; Barbosa, I.; Meyer, R.; Noda, A. y Sarduy, L. 2012. Criterios de bondad de ajuste en la selección de modelos no lineales en la descripción de comportamientos biológicos. Rev Cubana Cienc Agr, 46: 345-350.

Val J.E.; Freitas M.A.R.; Oliveira H.N.; Cardoso V.L.; Machado, P.F. e Paneto, J.C. 2004. Indicadores de desempenho em rebanho da raça Holandesa: curvas de crescimento e altura, características reprodutivas, produtivas e parâmetros genéticos. Arq Bras Med Vet Zootec, 56: 86-93. http://www.scielo.br/pdf/abmvz/v56n1/al 4v56nl. pdf (10/12/2008).

Vale, W.; Lourenzo, J. and Ohashi, O. 2002. Proceedings of the I Buffalo Symposium of Americas. PRODEPA - Governo do Estado do Pará. Belén, Brazil. 613 pp.

Verhulst, P.F. 1938. Notice sur la loi que la population poursuit dans son accroissement. Math Phy, 10: 113-121.

Von Bertalanffy, L.A. 1938. Quantitative theory of organic growth. Hum Biol, 10: 181-213. 